\title{
Innovaciones regulatorias del trabajo doméstico durante la pandemia en Argentina, Chile, Colombia, y Paraguay
}

[Regulatory innovations concerning domestic work during the pandemic in Argentina, Chile, Colombia, and Paraguay]

\author{
Loreto Poblete \\ Consejo Nacional de Investigaciones Científicas y Técnicas (CONICET) y \\ Universidad Nacional de San Martín, Argentina
}

\begin{abstract}
Resumen
Cuando comenzó la pandemia Covid-19 en marzo 2020, las trabajadoras del hogar se encontraban en una posición de vulnerabilidad debido a que la mayoría no tiene un contrato legal de trabajo y percibe ingresos relativamente bajos. Para algunas, su tiempo de trabajo se redujo, sus ingresos disminuyeron, y/o experimentaron suspensiones sin pago de salarios y despidos sin indemnizaciones. En ese contexto, los gobiernos buscaron desarrollar nuevas estrategias para garantizar los ingresos de las trabajadoras domésticas. Desde una perspectiva comparada, el artículo busca comprender la variedad de respuestas institucionales que se produjeron en cuatro países de América Latina (i.e., Argentina, Chile, Colombia y Paraguay). Basándose en el análisis de las regulaciones promulgadas durante los primeros nueve meses de la pandemia, el artículo analiza el carácter de las innovaciones en los marcos normativos específicos del trabajo doméstico remunerado y su carácter protector.
\end{abstract}

Palabras clave: trabajo doméstico, legislación laboral, seguridad social, pandemia Covid-19, cuarentena

\begin{abstract}
When the Covid-19 pandemic began in March 2020, domestic workers found themselves in a precarious position, as the majority earn low wages and do not hold formal contracts. Many saw their working hours cut and a reduction in income as a result. Some were left without wages as their positions were suspended, and others faced redundancy without compensation. In response, governments sought new strategies to safeguard the incomes of domestic workers. Adopting a comparative perspective, this article examines the various institutional solutions to the issue of paid domestic work applied in four Latin American countries-Argentina, Chile, Colombia, and Paraguay—during the Covid-19 pandemic. Taking into consideration all regulations implemented during the first nine months of the pandemic, the article offers an analysis of innovations to legal frameworks surrounding paid domestic work and their effectiveness in terms of protecting those at risk.
\end{abstract}

Keywords: paid domestic work, labor regulation, social security, Covid-19 pandemic, quarantine

Contacto: La comunicación sobre este artículo debe ser enviada a Loreto Poblete, email lorena.poblete@conicet.gov.ar

Financiamiento: La autora no declara fuentes específicas de financiamiento para este estudio, salvo el apoyo general del Consejo Nacional de Investigaciones Científicas y Técnicas (CONICET), dependiente del Ministerio de Ciencia de Argentina. 


\section{INTRODUCCIÓN}

En América Latina, el trabajado doméstico remunerado fue una de las categorías más afectadas por la crisis de la pandemia Covid-19. Esto se debe a las precarias condiciones de trabajo que experimentan las trabajadoras del hogar y al limitado alcance que tiene el marco legal en la práctica. La mayoría de las trabajadoras del hogar no cuentan con una relación laboral sujeta a un contrato formal de trabajo, por ello, su acceso a los derechos laborales y de la seguridad social es limitado. Esta informalidad condiciona el nivel de ingresos-en ocasiones, inferior al salario mínimo legal-y facilita el despido, ya que los empleadores no tienen que cumplir con el pago de indemnizaciones. Asimismo, la precariedad de sus condiciones laborales se relaciona con largas jornadas de trabajo y salarios pagados de manera irregular. Luego de la aprobación en 2011 del Convenio 189 (en adelante C189) de la Organización Internacional del Trabajo sobre trabajo decente en el contexto doméstico, 16 países latinoamericanos ratificaron el convenio y modificaron sus marcos legales siguiendo sus principios y lineamientos. El C189 propone concebir el trabajo doméstico como "un trabajo como ningún otro" y, al mismo tiempo, "un trabajo como cualquier otro" (Blackett, 1998, 2019; Organización Internacional del Trabajo, 2010). No obstante, la traducción del convenio en las regulaciones nacionales dio lugar a importantes diferencias en términos de protecciones. Actualmente, los marcos legales específicos del trabajo doméstico proveen niveles menores de protección en comparación con el régimen general aplicable a los asalariados y se observa un déficit de implementación de la normativa debido a la dificultad de inspeccionar el domicilio del empleador. En consecuencia, los derechos reconocidos en la ley no tienen aún un gran impacto en las prácticas cotidianas.

$\mathrm{Al}$ inicio de la pandemia Covid-19, en marzo 2020, las trabajadoras domésticas se encontraban en una posición muy vulnerable. Para algunas, su tiempo de trabajo se redujo y, por consiguiente, sus ingresos disminuyeron. Otras experimentaron suspensiones laborales sin pago de salarios y despidos sin indemnizaciones. Debido a que sólo las trabajadoras consideradas esenciales (i.e., a cargo de tareas de cuidado) estaban autorizadas a trabajar durante el confinamiento y, dado que las condiciones de vida y trabajo de los empleadores cambiaron radicalmente, la demanda de trabajo doméstico se redujo de manera significativa. En consecuencia, gran parte de las trabajadoras del hogar perdió total o parcialmente su fuente de ingresos. En este contexto, los gobiernos latinoamericanos tuvieron que desarrollar estrategias para garantizar los ingresos de las trabajadoras del hogar. Al igual que otros lugares, los países latinoamericanos adaptaron las instituciones preexistentes para enfrentar la crisis. El margen de innovación, particularmente en los países del Sur, fue muy limitado. Por lo tanto, el principal dilema de los gobiernos fue elegir entre aplicar las protecciones propias del marco legal que regula al trabajo doméstico remunerado, utilizar las políticas sociales vigentes, o combinar ambas estrategias.

Desde una perspectiva comparada, y analizando la normativa promulgada durante los primeros nueve meses de la pandemia en Argentina, Chile, 
Colombia, y Paraguay, este artículo buscó comprender cómo las características del marco legal, su nivel de implementación y la configuración del mercado condicionaron las respuestas en cada caso. Los casos de Argentina, Chile, Colombia y Paraguay resultan de particular interés al explorar la protección provista por los nuevos marcos legales durante la pandemia. Estos cuatro países ratificaron el C189 sólo algunos años después de su aprobación y modificaron sus marcos legales de acuerdo con sus disposiciones. Sin embargo, los marcos regulatorios nacionales presentan niveles de cobertura muy diferentes, además de niveles desiguales de implementación. Estos cuatro países muestran también diferencias respecto de la estructura y la evolución del sector. En 2019 en Argentina y Paraguay, las trabajadoras del hogar representan alrededor de $17 \%$ de las mujeres ocupadas, mientras que en Chile representan 7.9\% y, en Colombia, 6.7\%. Desde el 2000, el sector del trabajo doméstico se redujo a la mitad en Colombia y Chile. En Paraguay se mantuvo a los mismos niveles, y sólo en Argentina aumentó, pasando de $15.2 \%$ al $17.2 \%$ de las mujeres ocupadas (Valenzuela et al., 2020). Las modalidades de contratación contrastan entre estos países. En Colombia y Chile, cerca de 13\% de las trabajadoras se desempeñan "con cama", en Paraguay esta cifra es de 7.8\% y en Argentina 1.8\% (International Labour Organization [ILO], 2021). En consecuencia, estos cuatro países presentan diferentes arreglos institucionales y contextos específicos en los que puede evaluarse hasta qué punto las leyes laborales y de la seguridad social fueron capaces de proteger a las trabajadoras domésticas cuando la pandemia Covid-19 modificó abruptamente el funcionamiento del sector.

\section{Las protecciones disponibles}

En junio de 2011, la Conferencia Internacional del Trabajo adoptó C189 y la recomendación que lo acompaña (en adelante R201). Estos dos instrumentos proporcionan un modelo normativo innovador para el trabajo doméstico remunerado basado en los principios y derechos fundamentales del trabajo (Oelz, 2014; Tomei \& Belser, 2011): (a) la libertad de asociación y el derecho de negociación colectiva; (b) la abolición del trabajo infantil; (c) la eliminación de todas las formas de trabajo forzoso y; (d) la eliminación de la discriminación en el empleo y la ocupación. A fin de garantizar estos principios y derechos, el C189 propone protecciones contra el abuso, el acoso, la violencia y la discriminación; el derecho a ser informada de los términos y condiciones de empleo, incluida la ruptura de la relación laboral; el establecimiento de criterios claros para evitar jornadas laborales excesivas y disposiciones que rijan la periodicidad y las modalidades de pago, los períodos de descanso diarios y semanales, las vacaciones anuales pagas, la licencia por enfermedad, los períodos de prueba y el acceso a la seguridad social.

En América Latina, desde finales de la década de 1980, las organizaciones de trabajadoras del hogar se han movilizado fuertemente por el reclamo de derechos (Goldsmith et al., 2010), pero no se produjeron reformas importantes hasta después de la aprobación del C189. Paraguay modificó parcialmente el marco legal del trabajo doméstico en 1995, pero no se incorporaron nuevos derechos hasta dos décadas después. Argentina, que contaba con la legislación más antigua, no modificó el régimen laboral de 1956 hasta 2013, sin embargo, en 1999 
incorporó un régimen especial de seguridad social al marco legal existente. Colombia no reformó su legislación en materia de trabajo doméstico hasta 2012. Chile, en cambio, comenzó a reformar su marco legal en 1998, continuó entre 2007 y 2009, aprobó una ley integradora en 2014, y las últimas enmiendas tuvieron lugar en 2020. Por consiguiente, las innovaciones normativas propuestas por C189 fueron introducidas a las regulaciones nacionales siguiendo patrones diferentes.

\section{Marcos normativos disímiles}

Aunque estos países modificaron los marcos legales del trabajo doméstico remunerado siguiendo los lineamientos del C189, la cobertura varía considerablemente. Si bien los cuatro países reconocen el derecho al salario mínimo y al pago de horas extras, Colombia no incluyó el aguinaldo y autorizó el pago en especie de hasta $30 \%$ del salario. En cuanto al tiempo de trabajo, todos los países incorporaron a las trabajadoras domésticas en el régimen general de tiempo de trabajo, reconociendo los mismos límites a la jornada laboral, el descanso semanal y los feriados establecidos por el régimen general de trabajo. Asimismo, los distintos marcos normativos nacionales incluyeron vacaciones pagadas y licencias por enfermedad.

En cuanto a los principales beneficios de la seguridad social, las últimas reformas regulatorias incorporaron la licencia por maternidad, que fue una demanda de larga data de los sindicatos de trabajadoras del hogar. El seguro médico y las pensiones se incluyeron en todas las regulaciones nacionales. Sin embargo, la estructura de las contribuciones (i.e., la relación entre las contribuciones del empleador y de la trabajadora doméstica) limitan el tipo beneficios a los que las trabajadoras pueden acceder. En Argentina las trabajadoras domésticas que trabajan menos de 16 horas semanales para un mismo empleador deben realizar contribuciones suplementarias para poder utilizar el seguro de salud. En Paraguay, aun realizando aportes, quienes trabajan menos 12 horas por semana o menos de 48 horas mensuales para un único empleador no tienen derecho a percibir una jubilación. Entre los países bajo estudio, solamente Colombia reconocía el derecho al seguro de desempleo cuando comenzó la pandemia.

Modificar la estructura de las contribuciones en los sistemas de la seguridad social sigue siendo un desafío debido a dos cuestiones centrales: la inercia institucional -es decir, la dependencia de la trayectoria institucional (Mahoney, 2000)- y las características del empleador. Debido a que las instituciones de la seguridad social osificaron sus lógicas organizativas a lo largo del tiempo, los esfuerzos para adaptarlas a las especificidades del trabajo doméstico resultan problemáticos, particularmente debido a las distintas modalidades de contratación (e.g., con cama, sin cama, por horas, etc.). Y, dado que el empleador es un hogar, una familia, u otra mujer trabajadora, que no tiene la misma solidez económica que una empresa, las contribuciones de los empleadores establecidas en los marcos legales son menores, y por consiguiente, los beneficios también lo son. Durante los debates parlamentarios, los legisladores de estos países manifestaron que la ley debía adaptarse a la capacidad de cumplimiento de los empleadores. El principal argumento para reducir las contribuciones de los empleadores fue entonces la necesidad de 
garantizar la implementación de los nuevos marcos legales. Sin embargo, en un sector tradicionalmente caracterizado por la informalidad, la implementación de la nueva legislación sigue siendo deficitaria. Desde 2011, la formalización aumentó significativamente en la región debido a la introducción de innovaciones en los mecanismos de aplicación. En 2010, 42.3\% de las trabajadoras domésticas chilenas tenía un contrato formal de trabajo, 19.5\% en Argentina; 9.5\% en Colombia; y sólo $0.1 \%$ por ciento en Paraguay. En 2019, las trabajadoras formales representaban $50 \%$ en Chile, $26.4 \%$ en Argentina, $19.1 \%$ en Colombia y $5.1 \%$ en Paraguay (Valenzuela et al., 2020, p. 21).

A pesar del aumento del empleo formal, la informalidad persiste. Esto puede explicarse en parte por los obstáculos propios a la inspección del domicilio del empleador (Rodgers, 2009; Vega Ruiz, 2011) pero principalmente a la prevalencia de una noción de trabajo doméstico todavía muy cercana al modelo de servicio y servidumbre (Kuznesof, 1993; Valenzuela \& Mora, 2009). Dado que el trabajo doméstico es concebido como una actividad propia de las mujeres, las trabajadoras son consideradas como miembros de la familia y se encuentran sujetas a la autoridad familiar (Brites, 2007; Gorbán \& Tizziani, 2014). La desigualdad social que lleva a trabajadoras de sectores pobres, a menudo de comunidades racializadas, a buscar trabajo en hogares ricos refuerza este modelo. Así, debido a los altos niveles de informalidad, las trabajadoras domésticas no tienen acceso a los derechos laborales y los beneficios sociales vinculados al contrato de trabajo (Chen, 2011). Porque el Estado no puede garantizar el cumplimiento de la ley, los arreglos informales terminan dominando las relaciones laborales en este sector. Estas normas informales -es decir, la ley del hogar como lugar de trabajo (Blackett, 2019)- compiten, impugnan y resisten a las normas formales en la práctica cotidiana. En consecuencia, los marcos normativos jugaron un papel marginal en la protección de las trabajadoras domésticas durante la pandemia.

\section{Escenarios diversos}

Los efectos de la pandemia no fueron los mismos en todos los países bajo estudio. Según una encuesta realizada por la Federación Internacional de Trabajadoras del Hogar y la Confederación de Trabajadoras del Hogar de América Latina y el Caribe, entre abril y mayo de 2020 (Accari et al., 2021; Valenzuela \& Ramírez, 2021), la mayoría de las trabajadoras del hogar fueron despedidas o suspendidas en Colombia (77\%), más de la mitad fueron despedidas en Chile (56\%) y Paraguay (55\%), y sólo 15\% en Argentina. Alrededor del 20\% siguió trabajando con normalidad en Argentina y Chile, $17 \%$ en Paraguay y $8 \%$ en Colombia. La mitad de las trabajadoras del hogar en Argentina recibieron remuneraciones a pesar de que no poder ir a trabajar, en cambio en Chile y Colombia menos de $10 \%$ percibió su salario durante el mismo período (Accari et al., 2021).

La situación de las trabajadoras del hogar en relación con la pérdida de ingresos varía según el país. En Paraguay, casi un tercio de las trabajadoras se encontraban trabajando con reducción de ingresos. En Chile este grupo representaba 16\%, en Argentina $11 \%$ y 7\% en Colombia (Accari et al., 2021). Para $66.3 \%$ de trabajadoras argentinas, la pérdida de ingresos estaba relacionada con la disminución 
del tiempo de trabajo. Esta situación también fue sustancial en Chile (51.1\%) y Paraguay (45.1\%) donde gran parte de las trabajadoras vieron reducidas sus horas de trabajo. En contraste, en Colombia, solo 17.1\% perdió ingresos por esa razón. En algunos casos, los empleadores redujeron los salarios argumentando que, en razón de la inasistencia al lugar de trabajo a raíz del confinamiento, era razonable reducir las horas abonadas. En otros, cuando las trabajadoras se desempeñan por horas, la reducción del ingreso se debió a la pérdida de algunos puestos de trabajo (ILO, 2021).

En los países donde una mayor proporción de trabajadoras domésticas tiene un empleo formal, como en Chile y Argentina, la pérdida de empleo fue más pronunciada en los puestos informales. En Chile, se perdieron $43.4 \%$ de los puestos en el sector y el $63 \%$ de las trabajadoras informales fueron despedidas. De manera similar, en Argentina, donde se eliminó $37.3 \%$ de los puestos de trabajo y se perdió el 51.1\% de los trabajos informales (ILO, 2020). Por lo tanto, las posiciones formales parecen haber resistido mejor que los puestos informales a la crisis producida por la pandemia Covid-19. Las comparaciones entre países ilustran cuatro escenarios diferentes en los que los gobiernos desarrollaron distintas estrategias para proteger a las trabajadoras domésticas de la pérdida de ingresos durante los primeros nueve meses de pandemia. Estos escenarios están definidos por las características de los marcos legales de cada país, su nivel de implementación y la configuración del mercado laboral del sector al inicio de la crisis. En términos de marcos legales nacionales, Chile tiene un sistema de protección comparativamente más robusto y, en el extremo opuesto, Paraguay presenta un sistema más débil; Colombia y Argentina se sitúan en el medio, mostrando un nivel moderado de cobertura. Sin embargo, la implementación considerando los niveles de informalidad-condiciona la capacidad protectora de estos marcos legales. En Chile, la mitad de las trabajadoras del hogar pueden beneficiarse del alto nivel de cobertura del sistema de protecciones porque tienen contratos legales de trabajo. En el caso de Colombia, aunque el marco legal tiene una amplia cobertura en términos de provisiones, sólo una de cada cinco trabajadoras domésticas está protegida. En Argentina, donde los derechos laborales están garantizados pero el régimen de seguridad social para las trabajadoras del hogar otorga protecciones parciales, sólo una de cada cuatro trabajadoras está protegida por la ley. En contraste, en Paraguay, aunque la nueva normativa incluye muchas disposiciones del C189, la mayoría de las trabajadoras domésticas no están cubiertas por el marco legal debido al predominio del trabajo informal.

Si se tiene en cuenta la configuración del mercado laboral al inicio de la pandemia, la situación en Argentina parece menos crítica porque dos de cada diez trabajadoras del hogar continuaron trabajando con normalidad y la mitad del conjunto de las trabajadoras del hogar recibió sus salarios (o parte de ellos) durante el confinamiento. Sólo $11 \%$ perdió parte de su salario aun cuando trabajaba y $15 \%$ perdió ingresos por despido o suspensión. En Chile, por el contrario, menos de 30\% de las trabajadoras domésticas mantuvieron sus salarios independientemente de si trabajaron o no durante el confinamiento, $16 \%$ perdió parte de sus ingresos y $56 \%$ fue desvinculada. En Colombia, el mercado laboral del trabajo doméstico colapsó, dejando 77\% 
de las trabajadoras domésticas sin ingresos, $7 \%$ trabajando con pérdidas y solo $16 \%$ trabajando normalmente o recibiendo un pago durante la cuarentena. Finalmente, en Paraguay, debido a que la mayoría de las trabajadoras domésticas están empleadas de manera informal, los empleadores no pagaron los salarios durante la cuarentena. Sólo el 17\% trabajó regularmente, 50\% perdió su trabajo y casi una de cada tres experimentó una reducción en sus ingresos (Accari et al., 2021). Ante estos escenarios diferentes, los gobiernos de estos cuatro países buscaron desarrollar distintas estrategias para proporcionar ingresos a las trabajadoras del hogar que experimentaron una pérdida parcial o total de sus salarios durante los primeros meses de la pandemia de Covid-19.

\section{MÉTODO}

El objetivo de la investigación fue comprender de qué manera los gobiernos de Argentina, Chile, Colombia, y Paraguay utilizaron y adaptaron las herramientas legales disponibles para solucionar algunos de los problemas producidos por la pandemia Covid-19.

\section{Diseño de la investigación}

Por alcanzar los objetivos, se eligió la revisión documental de regulaciones como principal técnica de investigación debido al interés de estudiar las innovaciones regulatorias, particularmente, en momentos de crisis.

Esta investigación indaga sobre un proceso en curso, respecto del cuál es difícil precisar su culminación, por lo que en un primer momento se analizaron las regulaciones producidas durante los primeros seis meses (desde marzo hasta agosto 2020) dado que ese período resultó el más prolífero en cuanto al número de regulaciones como a la incorporación de innovaciones normativas. Luego, ese plazo se extendió tres meses para dar seguimiento a las políticas dirigidas específicamente a trabajadoras domésticas remuneradas. En este sentido, a pesar de que este trabajo ofrece una imagen parcial de las respuestas regulatorias producidas durante la pandemia Covid-19, ofrece un análisis exhaustivo de este período inicial.

\section{Muestra}

En esta investigación se trabajó con una compilación de 696 regulaciones -que incluye leyes, decretos presidenciales y parlamentarios, resoluciones de varios ministerios y protocolos- producidas en 14 países latinoamericanos durante los primeros nueve meses de la pandemia Covid-19 (de marzo a noviembre de 2020).

$\mathrm{Si}$ bien esta metodología permite comprender por qué, cómo, quién, y para quién se modificaron las normas durante la pandemia, no proporciona información sobre el modo en el que efectivamente se aplicaron o sobre los efectos de los cambios en las normas en la vida cotidiana. Es por ello que, adicionalmente, un corpus compuesto por 148 artículos de prensa fue utilizado para contextualizar las regulaciones. 


\section{Estrategia de análisis}

Para analizar este corpus todas las regulaciones fueron codificadas de acuerdo a cinco categorías: (a) regulaciones relativas a la restricción de la circulación (particularmente, las excepciones para los trabajadores esenciales); (b) políticas dirigidas a los trabajadores; (c) dispositivos para empresas; (d) medidas relativas a la seguridad social y; (e) políticas sociales.

Usando estas categorías, se analizó en detalle únicamente la legislación aplicable al trabajo doméstico remunerado y las normas relativas a la asistencia social que incluyen explícita o implícitamente a las trabajadoras domésticas. Una vez que se clasificó la legislación de cada país, se seleccionaron los cuatro países antes mencionados con el objeto de compararlos. La comparación se centró en el uso ad hoc y en las enmiendas realizadas en las regulaciones laborales, los beneficios de la seguridad social y las regulaciones relativas a transferencias directas de ingresos. Sólo se tuvieron en cuenta las reglamentaciones de nivel nacional porque la legislación laboral y de la seguridad social son competencia del gobierno nacional. Lo mismo ocurre con las políticas de transferencias directas de ingresos (al menos en los países bajo estudio).

\section{RESULTADOS}

La pandemia Covid-19 impuso a la urgencia como temporalidad exclusiva. Por consiguiente, los gobiernos se focalizaron exclusivamente en cambiar el uso de instituciones preexistentes y/o modificarlas marginalmente. A fin de analizar estas transformaciones el institucionalismo histórico ha develado el funcionamiento de distintos mecanismos de cambio institucional (Thelen, 2004). Entre ellos se destacan la superposición, la deriva y la conversión ya que permiten entender cómo cambian las instituciones cuando las reformas regulatorias son inconcebibles.

La superposición implica enmiendas, adiciones, o revisiones a un conjunto existente de instituciones. Estas enmiendas marginales producen alternaciones en la lógica originaria que estructura estas instituciones. La deriva ocurre cuando las instituciones o políticas se mantienen deliberadamente en su lugar mientras el contexto cambia. En este contexto, al modificarse altera los efectos de las instituciones que permanecen inalterables (Hacker et al., 2015). Finalmente, la conversión ocurre cuando las reglas permanecen formalmente iguales, pero se interpretan y aplican de manera novedosa (Hacker, 2005).

Durante la pandemia Covid-19, utilizando estos mecanismos de cambio institucional, cada uno de estos cuatro países intentó adaptar los recursos legales disponibles a fin de proteger a las trabajadoras del hogar. Cuando esos recursos resultaron insuficientes o inaplicables debido a los niveles de informalidad, las políticas de asistencia social -en particular, los programas de transferencias condicionadas de ingresoaparecieron como la única alternativa. 


\section{La adaptación temporaria de los marcos legales}

Como primera medida de contingencia, los gobiernos de los cuatro países establecieron importantes restricciones a la circulación a partir de marzo de 2020. Al principio, se presuponía que la cuarentena duraría uno o dos meses y, por consiguiente, algunos gobiernos decidieron que -aun si no todas las trabajadoras domésticas estaban autorizadas a trabajar- los salarios debían ser pagados regularmente por los empleadores. Como las medidas de aislamiento social obligatorio fueron extendiéndose sin una fecha límite clara, una primera estrategia fue modificar el uso de las protecciones legales vigentes. Dentro del marco normativo existen dos tipos de protecciones: las que dependen directamente del empleador (e.g., la licencia por enfermedad, los feriados, las vacaciones pagas) y las estructuradas a partir de seguros sociales (e.g., el seguro de salud, las pensiones, el seguro por desempleo). Con el objeto de mantener el pago de los salarios, algunos gobiernos decidieron reinterpretar la licencia por enfermedad y las vacaciones pagas, adaptándolas a la crisis producida por la pandemia Covid-19. Paraguay permitió que todos los empleados mayores de 65 años -exentos de ir a trabajar por ser considerados población en riesgo- hicieran uso de la licencia por enfermedad (Decreto 3.546, 21 de abril de 2020). Esta medida alcanzó a las trabajadoras del hogar a pesar de que la normativa relativa al trabajo doméstico remunerado en Paraguay no incluye este tipo de licencia. Colombia, por su parte, propuso utilizar las vacaciones pagas para proporcionar ingresos a quienes no pudieran asistir a sus lugares de trabajo debido a la vigencia del aislamiento social obligatorio. Con solo un día de anticipación, el empleador o el empleado podrían informar las fechas de las vacaciones -se tratara de vacaciones anuales anticipadas o acumuladas(Decreto 458, 27 de marzo de 2020). Esta medida general, también incluyó a las trabajadoras del hogar con contratos legales de trabajo. Mediante el mecanismo de conversión, los gobiernos de Paraguay y Colombia cambiaron el significado y el propósito de estos derechos. Originalmente, éstos eran el derecho a recuperarse de una enfermedad y el derecho al descanso anual, pero en esta coyuntura de emergencia, se utilizaron de manera ad hoc como derecho a percibir un salario a pesar de la inhabilitación para asistir al lugar trabajo. Si bien esta interpretación contradice el espíritu de la ley, estas medidas aparecieron como un compromiso entre la necesidad de percibir ingresos por parte de las trabajadoras del hogar y la responsabilidad de los empleadores de pagarles durante el confinamiento obligatorio. Sin embargo, debido a los altos niveles de informalidad en estos países, estas medidas no tuvieron mayor efecto.

Argentina, por su parte, adoptó un enfoque de carácter coercitivo. Inicialmente, el gobierno prohibió los despidos durante sesenta días (Decreto 329, 31 de marzo de 2020). Esta medida fue reconducida varias veces, $\mathrm{y}$ al momento de la escritura de este manuscrito, continuaba vigente (Decreto 39, 23 de abril de 2021). La normativa establece que si un trabajador es despedido mientras rige la prohibición de despido, tiene derecho a la doble indemnización. Al principio de la cuarentena, la mayoría de las trabajadoras domésticas recibían el pago de sus salarios con cierta regularidad. Sin embargo, con el paso del tiempo, esta medida creó una fuerte tensión entre las trabajadoras del hogar y sus 
empleadores. Debido a que muchos empleadores también habían perdido sus fuentes de ingresos a raíz del prolongado confinamiento que vivió el país, el pago regular de los salarios y las contribuciones patronales se volvió problemático. Además, cuando el gobierno estableció un subsidio dirigido a las empresas para que cubrieran parte de los salarios de sus empleados durante la pandemia (Decreto 332, 1 de abril de 2020), los empleadores de casas particulares no pudieron beneficiarse de esa ayuda gubernamental. Así, a través del mecanismo de superposición, el gobierno argentino extendió en el tiempo una medida extrema -utilizada sólo en situaciones críticas- $y$, al hacerlo, alteró la lógica protectora de la misma. En consecuencia, durante 2020 , se perdió $20 \%$ de los puestos en el sector. El trabajo doméstico fue el sector más afectado por la crisis resultante de la pandémica (Organización Internacional del Trabajo [OIT], 2020).

En dos de los países en los que el sistema de seguridad social incluye subsidio por desempleo, esta prestación se amplió durante la cuarentena, también mediante el mecanismo de superposición. En Colombia y Chile, el seguro de desempleo fue adaptado a fin de proporcionar ingresos a los trabajadores que habían sido suspendidos, despedidos, o que experimentaron una reducción en las horas de trabajo. Sin embargo, esta estrategia tuvo efectos ambivalentes ya que sólo $19 \%$ de las trabajadoras domésticas en Colombia tienen un contrato formal de trabajo y, en contraposición, la mitad de ellas tiene un empleo formal en Chile.

En Colombia, a las trabajadoras domésticas remuneradas se les otorgó el derecho al seguro por desempleo cuando el C189 fue incluido en la legislación nacional. La adaptación del seguro de desempleo durante la pandemia consistió en la flexibilización de las condiciones de acceso. Esto significó que quienes hubieran cotizado al sistema de seguridad social durante un año de manera continua o discontinua en los cinco años previos, podían recibir dos salarios mínimos legales divididos en tres cuotas consecutivas (Decreto 488, 27 de marzo de 2020). Posteriormente, en junio de 2020, el gobierno redujo el tiempo mínimo de cotización a seis meses para poder reclamar el derecho a la prestación por desempleo, en el caso de haber sido despedido después del 12 de marzo de 2020 (Decreto 801, 3 de junio de 2020).

En Chile, una nueva ley (Ley 21.227, 1 de abril de 2020) permitió el uso de las prestaciones por desempleo en caso de suspensión laboral durante la cuarentena. La ley establecía que si una empresa había sido fuertemente afectada por la crisis producida por la pandemia, el empleador y el trabajador podían firmar un pacto de suspensión temporal del contrato de trabajo. Este pacto sólo podía establecerse, durante los primeros seis meses de la pandemia, entre trabajadores que hubieran cotizado al sistema de seguridad social en los tres meses anteriores al pacto y al menos seis meses durante el último año. Si bien las trabajadoras del hogar no tenían derecho al seguro por desempleo antes de 2020, esta ley les permitió firmar un pacto de suspensión temporal del contrato de trabajo y recibir este subsidio especial por desempleo. Posteriormente, las trabajadoras domésticas fueron incorporadas al seguro general de desempleo (Ley 21.269, 11 de septiembre de 2020).

En síntesis, dado que gran parte del marco legal propio del trabajo doméstico remunerado en estos países no incluía disposiciones que permitieran 
proteger a las trabajadoras domésticas de la pérdida de ingresos antes de la pandemia de Covid-19, los gobiernos decidieron utilizar los mecanismos de conversión y de superposición a fin de adaptar las instituciones preexistentes a esta coyuntura sin precedentes. Solamente Chile pudo ir más allá de la coyuntura, introduciendo una enmienda al marco legal del trabajo doméstico remunerado.

\section{La asistencia social como único recurso}

Antes de la pandemia Covid-19, en un esfuerzo por contrarrestar los efectos de la informalidad en el sector, la mayoría de los países incluyeron a las trabajadoras domésticas en los programas de transferencias condicionadas de ingresos (PTC). En algunos casos, estos programas se integraron a la rama de beneficios no contributivos de la seguridad social, como en Argentina, donde la Asignación Universal por Hijo pasó a formar parte del régimen de Asignación Familiar (Ley 24.714, 16 de octubre de 1996). En otros casos, los PTC funcionaron como complemento de los bajos ingresos percibidos por trabajadores insertos en el sector informal. Estos programas, también llamados programas de corresponsabilidad, representan uno de los principales motores de innovación en el campo de la política social latinoamericana en las últimas dos décadas (Cecchini \& Atuesta, 2017). Tienen por objetivo a corto plazo, aliviar las condiciones de pobreza de los hogares más vulnerables y, a largo plazo, frenar la transmisión intergeneracional de la pobreza a través del desarrollo del capital humano (Cecchini \& Madariada, 2011). También promueven el empoderamiento femenino ya sea porque las mujeres son las que reciben las transferencias y son las responsables de cumplir con las condicionalidades, o porque se enfocan en lograr una mayor participación femenina en los diferentes niveles educativos (Lavinas, 2013). Las distintas variantes de los PTC se caracterizan por: (a) una transferencia mínima de ingresos monetarios -que puede estar acompañada por programas de capacitación o actividades de participación comunitaria-; (b) sujeta a condicionalidades -comúnmente, asistencia a establecimientos escolares y control de salud de niños y embarazadas- y; (c) dirigida a grupos específicos, clasificados como vulnerables tanto en términos económicos como sociales (Cecchini \& Vargas, 2014).

Desde marzo de 2020, todos los países considerados en este estudio utilizaron el mecanismo de cambio institucional denominado deriva con el fin de utilizar los PTC durante el período en el que los trabajadores informales no podían trabajar debido a las restricciones de circulación. En el contexto de crisis producido por la pandemia, las transferencias monetarias pasaron a ser el único ingreso de gran parte de los hogares en situación de pobreza, cuando en escenario prepandemia representaban sólo un ingreso adicional. Los gobiernos desarrollaron dos estrategias principales. Primero, los PTC suspendieron las condicionalidades. Por ejemplo, en Chile el Programa Seguridad y Oportunidades (Resolución 303 Exenta, 30 de mayo de 2020), y en Colombia el Programa Familias en Acción (Decreto 563, 15 de abril de 2020). En segundo lugar, se incrementaron temporalmente los montos de las transferencias monetarias. Tradicionalmente, las mismas eran consideradas un complemento de los ingresos dado que se presuponía que la participación en el mercado laboral-aun en posiciones informales 
y precarias con bajas remuneraciones- podía proporcionar ingresos de subsistencia (Velásquez Pinto, 2021). Este fue el caso de la Asignación Universal por Hijo en Argentina y del Tekopora en Paraguay (Ley 6.524, 1 de abril de 2020) donde se aumentaron los montos de las transferencias durante la pandemia. A pesar de que estas modificaciones tenían como objetivo cubrir a las poblaciones más vulnerables, muchas personas quedaron excluidas y sin ingresos durante la cuarentena. Por lo tanto, utilizando el mecanismo de superposición, se crearon nuevos programas de transferencias temporarias, sin condicionalidades sobre las estructuras institucionales de los programas vigentes. La mayoría de los programas estableció una transferencia única, que podía extenderse dentro de un período de tiempo limitado, generalmente hasta 90 días. Algunos programas incluían la posibilidad de ser renovados, mientras que otros no tenían cláusulas de reconducción. Dentro de los nuevos programas, algunos se enfocaron en poblaciones vulnerables previamente excluidas de los PTC, mientras que otros buscaron ampliar su alcance dando un carácter casi universal a las transferencias. Colombia, por ejemplo, estableció el Programa de Renta Solidaria como un programa temporal, luego reconducido durante 15 meses (Decreto 518, 20 de abril de 2020). En este caso, el objetivo fue brindar ingresos a aquellas poblaciones que, aunque se encontraban en situación de pobreza antes de la crisis producida por la pandemia Covid-19, no estaban incluidas en los PTC existentes.

Dentro de los programas de mayor cobertura, algunos buscaron aglutinar a los beneficiarios de los PTC existentes y a otras poblaciones vulnerables en un mismo programa, mientras que otros proponían crear una misma transferencia monetaria destinada a todos los hogares en situación de pobreza y a los trabajadores empobrecidos como consecuencia de la pandemia. Por ejemplo, Argentina estableció un programa de transferencias monetarias incondicionales, que también incluyó a los beneficiarios del PTC de mayor alcance -la Asignación Universal por Hijo- (Decreto 310, 24 de marzo de 2020). Esta transferencia, denominada Ingreso Familiar de Emergencia (IFE), estaba dirigida a trabajadores informales, trabajadores independientes formales de bajos ingresos, desempleados sin derecho a seguro de desempleo y trabajadoras del hogar. Este subsidio -que inicialmente comprendía un pago único- se extendió a cuatro cuotas durante los primeros nueve meses de la pandemia. Chile, por su parte, estableció un programa IFE, destinado a todos los trabajadores sin ingresos, originariamente con una duración de cuatro meses, pero con posibilidades de reconducción -incluso se planteó un IFE rebrote- (Ley 21.230, 16 de mayo de 2020). Debido a su amplia cobertura, la prensa lo describió como una ayuda para la clase media. En síntesis, los países de la región respondieron rápidamente utilizando la deriva como mecanismo de cambio institucional, a través de la expansión de los PTC existentes a fin de establecer transferencias temporarias incondicionales y, mediante el uso del mecanismo superposición, con la introducción de programas diseñados para cubrir una población más amplia, desde aquellos en pobreza extrema hasta clases medias empobrecidas. Las trabajadoras del hogar -en su mayoría trabajadoras informales en situación de pobreza- fueron incluidas en estos programas. Por lo tanto, fue a través de ellos que la mayoría de las trabajadoras del 
hogar en estos cuatro países pudieron tener acceso a ingresos básicos durante los primeros meses de la pandemia, desde marzo hasta noviembre de 2020 .

\section{CONCLUSIONES}

La pandemia Covid-19 creó un escenario crítico en el cual la efectividad del marco legal que protege a las trabajadoras domésticas fue puesto a prueba. Desde la aprobación del C189 en 2011, Argentina, Chile, Colombia, y Paraguay introdujeron importantes modificaciones a su legislación. La mayoría de los derechos laborales contenidos en la convención fueron incluidos en las leyes nacionales. Sin embargo, las disposiciones relativas a las prestaciones de la seguridad social (e.g., el seguro médico, las pensiones, o el seguro de desempleo) cuando se introdujeron, solo aportaban una cobertura parcial o deficiente. Las dificultades para modificar los esquemas de cotización clásicos de las instituciones de seguridad social para adaptarlos a las capacidades económicas del empleador dieron lugar a protecciones diferenciadas. La cobertura de los marcos legales resulta limitada porque la mayoría de las trabajadoras domésticas se encuentran en relaciones informales de trabajo. Si bien estos países desarrollaron formas innovadoras de implementar la legislación y cuentan con el activismo de las organizaciones de trabajadoras del hogar y otras ONG que promueven constantemente los derechos de las trabajadoras domésticas, la formalización sigue circunscrita a una minoría.

Cuando comenzó la pandemia, y muchas trabajadoras del hogar perdieron parcial o totalmente sus ingresos debido al confinamiento, los marcos legales no demostraron gran capacidad de protección frente a la pérdida de ingresos debido, por un lado, a sus disposiciones y, por otro, a su cobertura real. Así, los gobiernos comenzaron a improvisar utilizando mecanismos -como la superposición, la deriva o la conversión- para modificar marginal y circunstancialmente las instituciones laborales y de la seguridad social existentes. A pesar de que muchas regulaciones fueron promulgadas con la intención de reinventar las protecciones legales en un período de tiempo muy corto, en general, la situación crítica no apareció como una oportunidad para cuestionar las instituciones existentes y sus limitaciones, ni como una ocasión para introducir reformas más amplias.

Los cuatro países bajo estudio dan cuenta de escenarios diferentes. Chile, con un marco legal más robusto y la mitad de las trabajadoras domésticas con contratos formales de trabajo, pudo ampliar los beneficios del seguro de desempleo a la vez que los adaptó a este escenario particular y, al mismo tiempo, enmendó el marco normativo del trabajo del hogar. Este fue el único caso en el que la crisis producida por la pandemia Covid-19 condujo a reformas normativas. Por el contrario, en Argentina, que tiene un marco legal con una cobertura moderada, y donde sólo una de cada cuatro trabajadoras del hogar tiene una relación formal de trabajo, el gobierno decidió transferir el peso de la provisión de ingresos a los empleadores de casas particulares. Precisamente, prohibiendo los despidos e imponiendo 
el pago regular de los salarios, el gobierno colocó a las trabajadoras del hogar en una posición de gran vulnerabilidad porque quedaron completamente a merced de la decisión del empleador de respetar la ley. En el caso de Colombia, aunque el gobierno hizo modificaciones temporales a fin de utilizar de manera ad hoc ciertas disposiciones, el nivel de informalidad dejó estas medidas sin efecto. Finalmente, en Paraguay, donde la casi totalidad de las trabajadoras del hogar trabaja fuera del marco legal, el gobierno no pudo utilizar sus disposiciones para protegerlas de la pérdida de ingresos durante los primeros nueve meses de la pandemia.

En este escenario, los cuatro países ajustaron sus programas de transferencias monetarias vigentes para proveer ingresos a las trabajadoras domésticas. Si bien la utilización de las políticas de asistencia social, específicamente de los programas de transferencias directas de ingresos, podría aparecer como una opción coyuntural, la misma puso de manifiesto los límites de las protecciones legales. Después de una década de reformas normativas, los marcos legales relativos al trabajo doméstico remunerado en la región muestran claramente sus limitaciones a la hora de proteger a las trabajadoras del hogar frente a la pérdida de ingresos. Nuevamente la ley deja en evidencia su estrecho margen de intervención en una relación regida principalmente por la ley del hogar como lugar de trabajo. Si bien las reformas normativas en estos países buscaron deconstruir los cimientos de esta relación laboral necesitan abarcar e innovar más para poder contrarrestar la tendencia histórica a negar a las trabajadoras del hogar el derecho a ser consideradas verdaderas trabajadoras.

\section{REFERENCIAS}

Accari, L., Britez, J., \& Morales Pérez, A. (2021). Right to health, right to live: Domestic workers facing the COVID-19 crisis in Latin America. Gender \& Development, 29(1), 11-33. https://doi.org/10.1080/13552074.2021.1885213

Blackett, A. (1998). Making domestic work visible: The case for specific regulation (Working paper $\mathrm{N}^{\circ} 2$ ). Labour Law and Labour Relations Programme. https://www.ilo.org/public/libdoc/ilo/1998/98 B09_500_engl.pdf

Blackett, A. (2019). Everyday transgressions: Domestic workers' transnational challenge to international labor law. Cornell University Press.
Brites, J. (2007). Afeto e desigualdade: Gênero, geração e classe entre empregadas domésticas e seus empregadores. Cadernos Pagu, (29), 91-109. https://doi.org/10.1590/S0104-83332007000200005

Cecchini, S., \& Atuesta, B. (2017). Programas de transferencias condicionadas en América Latina y el Caribe. Tendencias de cobertura e inversión (Documento de trabajo, serie políticas sociales, $\mathrm{N}^{\circ} 224$, LC/TS.2017/40). CEPAL. https://bit.ly/3EBar4W

Cecchini, S., \& Madariaga, A. (2011). Programas de transferencias condicionadas. Balance de la experiencia reciente en América Latina y el Caribe. (Documento de trabajo, serie cuadernos 

trabajo doméstico durante la pandemia en Argentina, Chile, Colombia, y Paraguay. Revista de Sociología, 36(2), 34-49. https://doi.org/10.5354/0719-529X.2021.65569

de la CEPAL N 95, LC/G.2497-P). CEPAL. https://repositorio.cepal.org/handle/11362/27854

Cecchini, S., \& Vargas, L. H. (2014). Transferencias de ingresos para la erradicación de la pobreza. Dos décadas de experiencia en los países de la Unión de Naciones Suramericanas (UNASUR) (Documento de trabajo, LC/L.3925). CEPAL; UNASUR. https://bit.ly/3Et68bM

Chen, M. (2011). Recognizing domestic workers, regulating domestic work: Conceptual, measurement, and regulatory challenges. Canadian Journal of Women and the Law, 23, 167-184.http://doi.org/10.3138/cjwl.23.1.167

Goldsmith, M. R., Canedo, R. B, Ferrari, A., \& Vence, M. C. (2010). Hacia un fortalecimiento de derechos laborales en el trabajo de hogar: Algunas experiencias de América Latina. Friedrich-Ebert-Stiftung.

Gorbán, D., \& Tizziani, A. (2014). Inferiorization and deference: The construction of social hierarchies in the context of paid domestic labor. Women's Studies International Forum, 46, 54-62. https://doi.org/10.1016/j.wsif.2014.01.001

Hacker, J. S. (2005). Policy drift: The hidden politics of US welfare state retrenchment. En W. Streeck \& K. Thelen (Eds.), Beyond continuity: Institutional change in advanced political economies (pp. 40-82). Oxford University Press.

Hacker, J. S., Pierson, P., \& Thelen, K. (2015). Drift and conversión: Hidden faces of institutional change. En J. Mahoney, \& K. Thelen (Eds.) Advances in comparative- historical analysis (pp. 180-208). Cambridge University Press.

International Labour Organization. (2021). Making decent work a reality for domestic workers. Progress and prospects ten years after the adoption of the Domestic Workers Convention, 2011 (Documento de trabajo, $\mathrm{N}^{\circ}$ 189). https://bit.ly/2ZQuwoT

Kuznesof, E. (1993). Historia del servicio doméstico en la América hispana (14921980). En E. Chaney \& M. Garcia Castro (Eds.), Muchacha, cachifa, criada, empleada, empregadinha, sirvienta y... nada más. Trabajadoras del hogar en América Latina y el Caribe (pp. 25-40). Nueva Sociedad.

Lavinas, L. (2013). 21st century welfare. New Left Review, 84, 5-40. https://bit.ly/3bwX4pJ

Mahoney, J. (2000). Path dependence in historical sociology. Theory and Society, 29(4), 507-548. https://www.jstor.org/stable/3108585

Oelz, M. (2014). The ILO's domestic workers convention and recommendation: A window of opportunity for social justice. International Labour Review, 153(1), 143-172.

https://doi.org/10.1111/j.1564-913X.2014.00200.x Organización Internacional del Trabajo. (2010). Trabajo decente para los trabajadores domésticos. (Informe IV (1) de la Conferencia Internacional del Trabajo, sesión 99\%). https://bit.ly/3pVuEOz

Rodgers, J. (2009). Cambios en el servicio doméstico en América Latina. En M. E. Valenzuela \& C. Mora (Eds.), Trabajo doméstico: Un largo camino hacia el trabajo decente (pp. 71-114). 
Oficina Internacional del Trabajo. https:/bit.ly/3mzfnAR

Thelen, K. (2004) How institutions evolve: The political economy of skills in comparativehistorical perspective. Cambridge University Press.

Tomei, M., \& Belser, P. (2011). New ILO standards on decent work for domestic workers: A summary of the issues and discussions. International Labour Review, 1503-4), 431-438. https://doi.org/10.1111/j.1564-913X.2011.00127.x

Valenzuela, M. E., \& Mora, C. (Eds.) (2009). Trabajo doméstico: Un largo camino hacia el trabajo decente. Oficina Internacional del Trabajo. https://bit.ly/3mzfnAR

Valenzuela, M. E., \& Ramírez, A. P. (2021). The impact of Covid-19 on domestic workers in Latin America. International Domestic Workers Federation. https://bit.ly/3nRFkuT Valenzuela, M. E., Scuro, M. L., \& Vaca-Trigo, I. (2020). Desigualdad, crisis de los cuidados y
Poblete, L. (2021). Innovaciones regulatorias del trabajo doméstico durante la pandemia en Argentina, Chile, Colombia, y Paraguay. Revista de Sociología, 36(2), 34-49. https://doi.org/10.5354/0719-529X.2021.65569

migración del trabajo doméstico remunerado en América Latina [Serie asuntos de género, $\mathrm{N}^{\circ} \quad 158, \quad$ LC/TS.2020/179]. CEPAL. https://repositorio.cepal.org/bitstream/handl e/11362/46537/1/S2000799_es.pdf

Vega Ruiz, M. L. (2011). L'administration et l'inspection du travail dans le domaine du travail domestique: Les expériences de l'Amérique Latine. Canadian Journal of Women and the Law, 23(1), 341-358. https://doi.org/10.3138/cjwl.23.1.341

Velásquez Pinto, M. (2021). La protección social de los trabajadores informales ante los impactos del COVID-19 [Documento de proyectos (LC/TS.2021/37)]. CEPAL. https://bit.ly/3jXiBwF

Manuscrito recibido: 21-08-2021 Manuscrito aceptado: 19-10-2021 